\title{
Optimizing working space in laparoscopy: CT-measurement of the effect of neuromuscular blockade and its reversal in a porcine model
}

\author{
John Vlot • Patricia A. Specht · René M. H. Wijnen • \\ Joost van Rosmalen • Egbert G. Mik • \\ Klaas M. A. Bax
}

Received: 22 April 2014/Accepted: 27 September 2014/Published online: 1 November 2014

(C) Springer Science+Business Media New York 2014

\begin{abstract}
Objective The objective of this paper was to determine the effect of neuromuscular blockade (NMB) on working space in a porcine laparoscopy model.

Background Conflicting results on the effect of NMB on laparoscopic working space are found in literature. Almost all studies are limited by absence of objective assessment of working space or use surrogate outcomes.

Methods In a standardized porcine laparoscopy model, laparoscopic working-space dimensions with and without NMB were investigated in 16 animals using computed tomography at intra-abdominal pressures of $0,5,10$, and $15 \mathrm{mmHg}$ during multiple runs of abdominal insufflation. Results No statistically significant effect of NMB on abdominal dimensions and laparoscopic working-space volume was found during $\mathrm{CO}_{2}$ pneumoperitoneum. In contrast, the effect of pre-stretching of the abdominal wall by a previous abdominal insufflation was found to be significant. Conclusions This experimental study confirms the results from several clinical studies that NMB does not influence laparoscopic working space. Studies dealing with working space during laparoscopy should take note of pre-stretching bias.
\end{abstract}

J. Vlot $(\bowtie) \cdot$ R. M. H. Wijnen · K. M. A. Bax

Department of Pediatric Surgery, Erasmus MC: University

Medical Center, P.O Box 2060, 3000 CB Rotterdam,

The Netherlands

e-mail: john.vlot@erasmusmc.nl

P. A. Specht · E. G. Mik

Department of Anesthesiology, Erasmus MC: University

Medical Center, Rotterdam, The Netherlands

J. van Rosmalen

Department of Biostatistics, Erasmus MC: University Medical

Center, Rotterdam, The Netherlands
Keywords Working space - Pneumoperitoneum . Neuromuscular blockade $\cdot$ Animal model $\cdot \mathrm{CO}_{2}$. Laparoscopy

Optimizing working space is essential for safe and efficient minimal access surgery (MAS) [1-4]. It seems logical to assume that decreasing muscle tone by neuromuscular blockade (NMB) will have a positive effect on working space during laparoscopic surgery. However, the available literature on this subject is scarce and does not support this assumption, neither experimentally [5] nor clinically [6-8]. Almost all clinical studies used surgeon's subjective assessment of working space [6-10] or the duration of surgery [7-11] as endpoints. In addition, the level of neuromuscular block was not well documented in some of the studies [7, 8]. As working space in laparoscopic surgery is strongly related to the age/size of the patient, it becomes more critical in small children [12]. Even a small gain in working space can markedly improve surgical conditions in this patient group. To measure the effect of various interventions on laparoscopic working space, we have previously developed a porcine model with standardized anesthesiologic and surgical techniques [13]. Computed tomography (CT) was used to accurately measure laparoscopic working-space dimensions. The present study evaluates the effect of NMB and its reversal on laparoscopic working-space dimensions in this animal model.

\section{Methods}

Animals

Sixteen juvenile Landrace pigs, weighing approximately $20 \mathrm{~kg}$, were studied. For reasons of homogeneity, we chose to study only female animals. Approval was obtained from the institutional animal ethics committee. 
Anesthesia

Pigs were subjected to a similar anesthesiologic protocol as used in prior experiments [13]. In short, premedication consisted of intra-muscular midazolam $(1 \mathrm{mg} / \mathrm{kg})$ and ketamine $(30 \mathrm{mg} / \mathrm{kg})$. After cannulation of an auricular vein, anesthesia was induced with propofol $(1.5 \mathrm{mg} / \mathrm{kg})$ and sufentanil $(5 \mathrm{mcg} / \mathrm{kg})$. Next, tracheotomy was performed. Anesthesia was maintained with intravenous sufentanil $(4 \mathrm{mcg} / \mathrm{kg} / \mathrm{h})$ and propofol $(8 \mathrm{mg} / \mathrm{kg} / \mathrm{h})$. Mechanical ventilation was volume-controlled $(10 \mathrm{ml} / \mathrm{kg})$ with a positive end expiratory pressure (PEEP) of $5 \mathrm{cmH}_{2} \mathrm{O}$. Tidal volumes were kept constant, respiratory rate was adjusted to maintain end-tidal $\mathrm{CO}_{2}\left(\mathrm{ETCO}_{2}\right)$ between 4.5 and $7.0 \mathrm{kPa}$. Vascular access to the carotid artery and internal jugular vein was established. Regular sampling of blood for hematocrit and blood gas analysis was done. Core temperature, heart rate (HR), mean arterial blood pressure (MAP), respiratory rate (RR), peak inspiratory airway pressure (PIP), and $\mathrm{ETCO}_{2}$ were measured continuously. Normothermia was maintained during the experiments using an electric heating blanket.

\section{$\mathrm{CO}_{2}$ pneumoperitoneum and $\mathrm{CT}$ scanning}

Here also, a technique similar to the one used in prior experiments was used [13]. In short, a radially expanding trocar (VersaStep ${ }^{\mathrm{TM}}$, Covidien, Dublin, Ireland) was placed in the midline a few centimeter above the umbilicus. The correct intra-abdominal position was verified endoscopically. When hemodynamic and respiratory parameters were stable, pigs were transported from the laboratory facility to the CT scanner. After the pig was installed on the scanning tray, an electronic $\mathrm{CO}_{2}$ insufflator was attached to the abdominal trocar. Abdominal $\mathrm{CO}_{2}$ insufflation with a stepwise increase of IAP from 0 to 5,10 , and $15 \mathrm{mmHg}$ was performed (insufflation-run). At each level of intra-abdominal pressure (IAP), a 5 min waiting period was taken into account for stabilization of blood pressure, PIP and $\mathrm{ETCO}_{2}$. Thorax and abdomen were then scanned. To minimize respiratory motion artifacts, scans were made during an expiratory-hold maneuver while maintaining PEEP at $5 \mathrm{cmH}_{2} \mathrm{O}$. Scanning at each IAP level took approximately $5 \mathrm{~s}$. Pigs were sacrificed after completion of all scans.

\section{Neuromuscular blockade}

In all animals, neuromuscular function was monitored continuously by acceleromyography at the quadriceps femoris muscle using the TOF Guard (Organon Teknika NV, Turnhout, Belgium). The femoral nerve was stimulated using surface pediatric electrodes. After stabilization and calibration of the train of four (TOF) signal, repetitive TOF stimulation was performed every $15 \mathrm{~s}$, using supra-maximal stimuli of $2 \mathrm{~ms}$. The TOF ratio is the height of the fourth twitch, compared to the first twitch height (T4/T1). During deep NMB, there is no response to TOF stimulation. When all four responses to TOF stimulation are present and the TOF ratio is $>90 \%, \mathrm{NMB}$ is considered to be fully recovered [14]. Rocuronium was used for muscle paralysis. A bolus of $1.4 \mathrm{mg} / \mathrm{kg}$ $\left(2 \times \mathrm{ED}_{90}\right.$, i.e., twice the effective dose at which $90 \%$ of subjects in the pig population is paralyzed) was followed by continuous administration of rocuronium $4 \mathrm{mg} / \mathrm{kg} / \mathrm{hour}$ under TOF guidance. When T1 reappeared, another bolus of rocuronium $\mathrm{ED}_{90}$ was administered. For reversal of NMB, sugammadex $(4 \mathrm{mg} / \mathrm{kg})$ was used. Its effect on neuromuscular function was also monitored with the TOFGuard.

By predetermined randomization, half of the animals (group A) received no NMB during the first and second run of stepwise abdominal insufflation up to an IAP of $15 \mathrm{mmHg}$. In this way, the effect of pre-stretching of the abdominal wall on working-space dimensions could be measured. After NMB, a third insufflation-run was performed and after reversal of NMB a fourth one. This was done to measure the additional effect of NMB and its reversal on working-space dimensions.

In the other eight animals (group B), NMB was attained prior to the first insufflation-run and maintained during the second insufflation-run. A third insufflation-run was performed after reversal of NMB.

\section{Outcome measures}

To establish homogeneity of the animals, body weight and total length of the first five lumbar vertebrae were measured [15]. Core temperature, HR, MAP, RR, PIP, ETCO ${ }_{2}$, and TOF were recorded at each level of IAP. Pneumoperitoneum volumes and working-space linear dimensions (Fig. 1) were measured in Osirix ${ }^{\circledR}$ using a dataset of 1-mm slices [16]. With the definition of appropriate thresholds, semiautomatic detection of $\mathrm{CO}_{2}$ in the abdomen could be done on transverse slices, which could be integrated to a total volume of $\mathrm{CO}_{2}$ pneumoperitoneum [17]. All volumes were visually checked for inadvertent inclusion of gas in the bowel. For the linear dimensions, maximal internal antero-posterior diameter of the abdomen (anterior peritoneal lining to the anterior vertebral column) and maximal internal transverse diameter were measured in a transverse plane at the level of the umbilicus. The maximal distance between the upper border of the pubic symphysis and the highest diaphragmatic peritoneal lining was measured in a mid-sagittal plane (Fig. 1). 


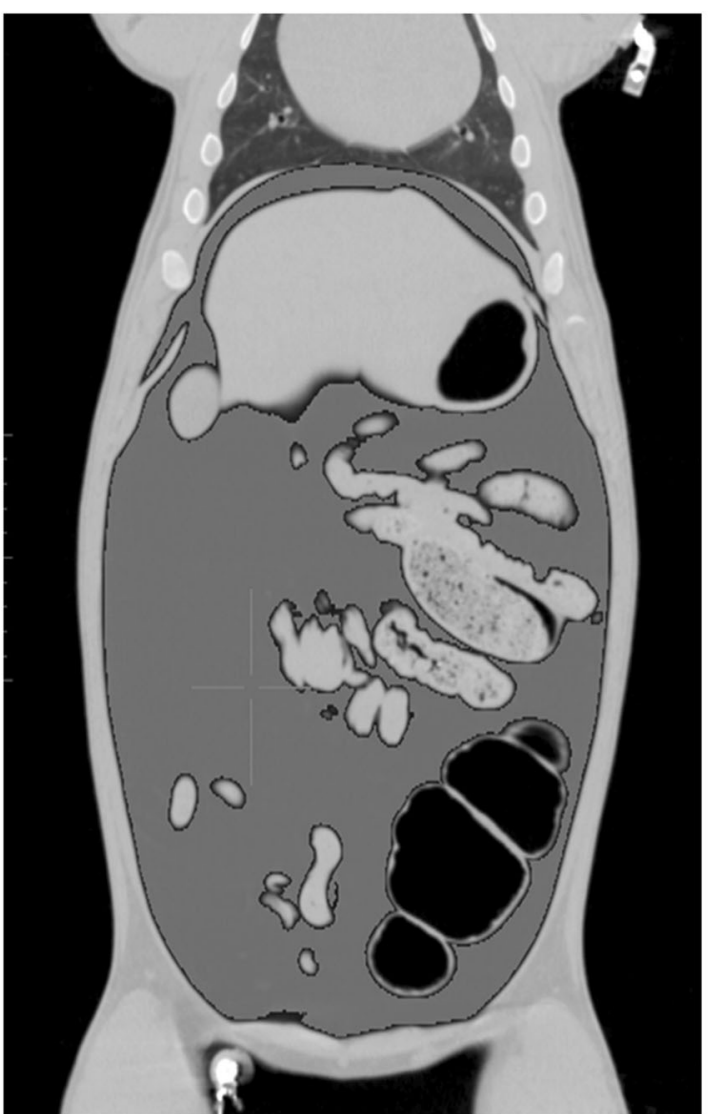

$\mathrm{CO}_{2}$ pneumoperitoneum volume (dark grey area)

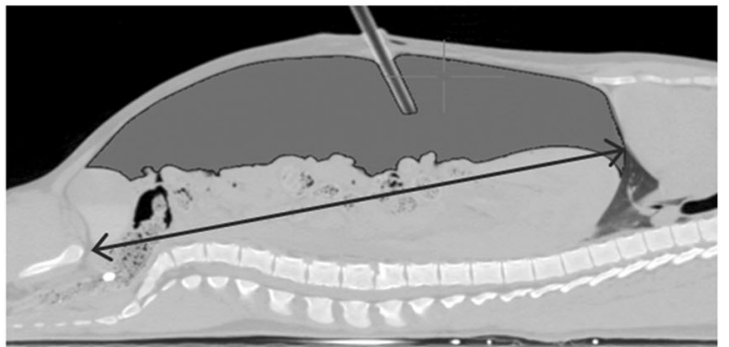

Symphysis to diaphragm

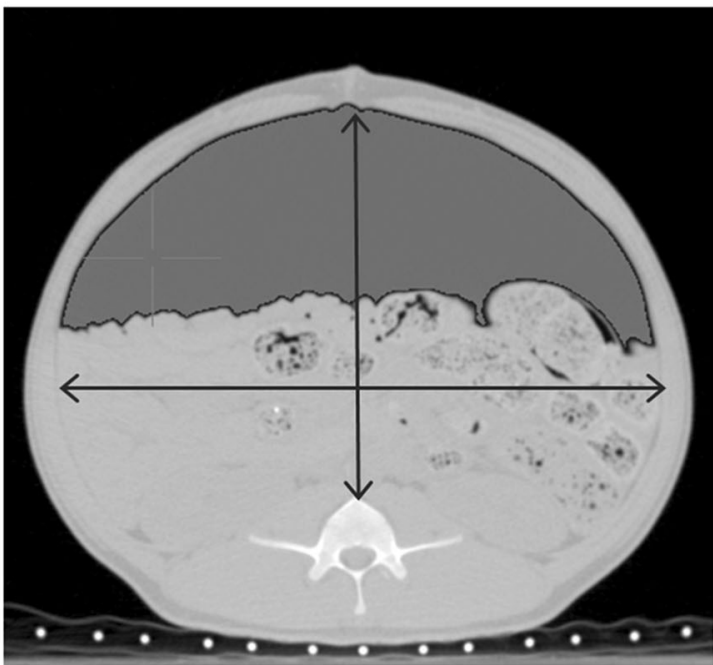

Internal AP and transverse diameter

Fig. 1 CT-images and reconstructions showing working-space dimensions. AP antero-posterior (anterior peritoneal lining to the anterior vertebral column)

\section{Statistics}

Normality of the volume and cardiorespiratory data was confirmed by visual assessment and using a Shapiro-Wilk test. Data are presented as means with standard deviations (SDs), except for the intra-abdominal $\mathrm{CO}_{2}$ volumes where standard errors of the mean (SEM) were used. Differences between groups in body weight, lumbar length, and cardiorespiratory data were assessed using independent samples $t$ tests. Differences in cardiorespiratory data within the same animal were assessed using paired samples $t$ tests. A two-tailed $p$ value of $<.05$ was considered significant. To estimate the effects of IAP, pre-stretching, and NMB on working-space volume, a linear mixed model was used. In this analysis, only the data from the first and second insufflation-run were used. This was done for reasons of homogenicity, hereby excluding the unknown additional effect of multiple runs of insufflation. In the linear mixed model, the main effects of pressure, pre-stretching, and NMB were included as independent variables. Two-way interaction effects of IAP, pre-stretching, and NMB were added to the model if the effect was statistically significant.
All independent variables were treated as categorical variables. To account for the within-subject correlations, a random intercept and a random main effect of IAP were included for each pig.

\section{Results}

Homogeneity of the pigs

Mean body weight for all pigs was $23.2 \mathrm{~kg}$ (SD 1.28). Mean length of the first five lumbar vertebrae was $11.8 \mathrm{~cm}$ (SD .33). No statistically significant differences in body weight and lumbar length existed between the animals in group $\mathrm{A}$ and $\mathrm{B}$.

\section{Cardiorespiratory parameters}

Values for all cardiorespiratory parameters during the first insufflation-run are shown in Table 1. A statistically significant increase in PIP and $\mathrm{ETCO}_{2}$ from baseline (IAP $=0 \mathrm{mmHg}$ ) occurred during the first insufflation-run 
Table 1 Cardiorespiratory parameters during the first insufflationrun (mean values)

\begin{tabular}{lllllllll}
\hline IAP & 0 & & 5 & & 10 & & 15 & \\
NMB: & - & + & - & + & - & + & - & + \\
\hline MAP & 72 & 77 & 75 & 90 & $76^{\mathrm{a}}$ & $97^{\mathrm{a}}$ & $76^{\mathrm{b}}$ & $95^{\mathrm{b}}$ \\
$\mathrm{HR}$ & 83 & 88 & 78 & 85 & 81 & 81 & 80 & 83 \\
$\mathrm{RR}$ & 26 & 24 & 26 & 24 & 26 & 24 & 28 & 24 \\
$\mathrm{PIP}$ & 20 & 20 & 20 & 20 & 24 & 24 & 30 & 29 \\
$\mathrm{ETCO}_{2}$ & 5.3 & 5.7 & 5.5 & 6 & 5.9 & 6.4 & 5.9 & 6.6 \\
\hline
\end{tabular}

$I A P$ intra-abdominal pressure $(\mathrm{mmHg}), N M B$ neuromuscular blockade, $A P$ antero-posterior, $M A P$ mean arterial blood pressure $(\mathrm{mmHg})$, $H R$ heart rate (beats/min), $R R$ respiratory rate (breaths/min), $P I P$ peak inspiratory airway pressure $\left(\mathrm{cmH}_{2} \mathrm{O}\right), E T C \mathrm{O}_{2}$ end-tidal $\mathrm{CO}_{2}(\mathrm{kPa})$

${ }^{\mathrm{a}} p=.038$

${ }^{\mathrm{b}} p<.01$ (independent samples $t$ test)

at an IAP greater than $10 \mathrm{mmHg}(p=<.01$ in both group $\mathrm{A}$ and $\mathrm{B}$ at IAPs of 10 and $15 \mathrm{mmHg}$ ). A minor increase in $\mathrm{RR}(p=.197)$ was necessary at the end of the first insufflation-run to maintain $\mathrm{ETCO}_{2}$ below $7 \mathrm{kPa}$ in group A at an IAP of $15 \mathrm{mmHg}$. During the first insufflation-run, MAP increased in both groups when compared to baseline. In group $\mathrm{A}$, the increase was not statistically significant. In group B, however, MAP showed a statistically significant increase during the first insufflation-run $(p<.01$ at all IAPs $>0$ ). When comparing the effect of IAP on MAP between groups $\mathrm{A}$ and $\mathrm{B}$, statistically significant differences existed during the first insufflation-run at an IAP of $10(p=.038)$ and $15 \mathrm{mmHg}(p<.01)$. The effects found during the second insufflation-run were similar (data not shown). No statistically significant other changes were found.

\section{Neuromuscular blockade}

In group A, TOF ratio was always above $91 \%$ before $\mathrm{NMB}$, below $4 \%$ with $\mathrm{NMB}$, and above $99 \%$ after reversal of NMB. In animals in group B, TOF ratio was always below $5 \%$ with NMB and above $92 \%$ after its reversal.

$\mathrm{CO}_{2}$ pneumoperitoneum (working-space dimensions)

The observed mean $\mathrm{CO}_{2}$ pneumoperitoneum volumes during the insufflation-runs for group $\mathrm{A}$ and $\mathrm{B}$ are shown in Table 2. In the mixed model analysis, the effects of IAP and pre-stretching as well as the interaction effect of IAP and pre-stretching were statistically significant $(p<.01)$.

The estimated effect of NMB was not statistically significant (value $196 \mathrm{ml}, p=.495,95 \%$ confidence interval -404 to $796 \mathrm{ml}$ ). The effect of pre-stretching, however, was highly significant $(p<.01)$.
In group $\mathrm{A}$, pre-stretching resulted in an increase in mean $\mathrm{CO}_{2}$ pneumoperitoneum volume of $21 \%$ at a repeat IAP of $5 \mathrm{mmHg}, 7 \%$ at a repeat IAP of $10 \mathrm{mmHg}$, and $3 \%$ at a repeat IAP of $15 \mathrm{mmHg}$. In group $\mathrm{B}$, mean $\mathrm{CO}_{2}$ pneumoperitoneum volume showed an increase during the second insufflation-run of $19 \%$ at a repeat IAP of $5 \mathrm{mmHg}, 8 \%$ at a repeat IAP of $10 \mathrm{mmHg}$, and $4 \%$ at a repeat IAP of $15 \mathrm{mmHg}$.

A third and fourth insufflation-run with addition and/or reversal of NMB mostly showed a small further increase in mean $\mathrm{CO}_{2}$ pneumoperitoneum volume with a small decrease only at an IAP of $5 \mathrm{mmHg}$ in group A (Table 2).

Mean internal working-space distances at all IAP levels during the first insufflation-run are shown in Fig. 2. None of the linear dimensions showed a statistically significant difference between group A (no-NMB) and group B (NMB) in the first or second insufflation-run (data not shown).

\section{Discussion}

It seems logical to assume that NMB increases working space during laparoscopy. This assumption, however, has not been endorsed by the literature. In a study of pigs, Chassard et al. did not find a positive effect of muscle paralysis on working space [5]. Also in a clinical study in young, non-obese gynecologic patients, Chassard et al. could not demonstrate a positive effect [6]. In the guidelines on pneumoperitoneum of the European Association for Endoscopic Surgery, nothing is written about NMB [18]. The subject of NMB during laparoscopic surgery has re-emerged in recent studies. Staehr-Rye et al. plan to investigate the correlation between the level of muscle paralysis and the surgeon's interpretation of working space [10]. This and most other studies use postoperative surgeon's questionnaires on adequacy of exposure as an endpoint $[6,9,10]$. The robustness of such subjective evaluations can be questioned. Lindekaer et al. used the distance from the trocar entrance to the promontory as a more objective measure of working-space dimensions [19]. However, measuring the trocar distance before and after NMB implies two measurements, inevitably introducing a confounding effect of pre-stretching of the abdominal wall [20]. Although no quantitative data on this pre-stretching effect are available in human laparoscopy, we believe that it should be taken into consideration when interpreting the results of studies dealing with laparoscopic working space.

Our porcine laparoscopy model, as used in prior experiments to investigate the effect of IAP, mechanical bowel preparation, and pre-stretching of the abdominal wall, abided by strict anesthesiologic and surgical protocols $[13,20,21]$. In this way, the effect of NMB per se could be 
Table 2 Mean volumes of $\mathrm{CO}_{2}$ pneumoperitoneum in milliliters (SEM)

$n$ number of animals,

$S E M$ standard error of the mean, $I A P$ intra-abdominal pressure, $N M B$ neuromuscular blockade, $\mathrm{mmHg}$ millimeters of mercury

Fig. 2 Working-space linear dimensions $(\mathrm{cm})$ during the first insufflation-run (mean values). No statistically significant differences exist between group A (no-NMB) and group B (NMB). IAP intra-abdominal pressure. NMB neuromuscular blockade. AP antero-posterior

\begin{tabular}{|c|c|c|c|c|c|}
\hline \multirow{2}{*}{$\begin{array}{l}\text { Group A } \\
n=8\end{array}$} & \multirow[b]{2}{*}{ IAP $(\mathrm{mmHg})$} & \multicolumn{4}{|c|}{ Insufflation-run $\rightarrow$} \\
\hline & & $\begin{array}{l}\text { 1st } \\
\text { no-NMB }\end{array}$ & $\begin{array}{l}\text { 2nd } \\
\text { no-NMB }\end{array}$ & $\begin{array}{l}3 \mathrm{rd} \\
\mathrm{NMB}\end{array}$ & $\begin{array}{l}\text { 4th } \\
\text { Reversal }\end{array}$ \\
\hline & 0 & 0 & 0 & 0 & 0 \\
\hline & 5 & $1,403(183)$ & $1,691(220)$ & $1,808(210)$ & $1,774(235)$ \\
\hline & 10 & $2,628(235)$ & $2,819(263)$ & $2,930(266)$ & $2,979(282)$ \\
\hline & 15 & $3,189(262)$ & $3,290(271)$ & $3,369(281)$ & $3,459(284)$ \\
\hline \multicolumn{2}{|l|}{ Group B } & \multicolumn{3}{|c|}{ Insufflation-run $\rightarrow$} & \\
\hline \multirow[t]{5}{*}{$n=8$} & IAP $(\mathrm{mmHg})$ & $\begin{array}{l}1 \mathrm{~s} \\
\mathrm{~N}\end{array}$ & & $\begin{array}{l}\text { 2nd } \\
\text { NMB }\end{array}$ & $\begin{array}{l}\text { 3rd } \\
\text { Reversal }\end{array}$ \\
\hline & 0 & & 0 & 0 & 0 \\
\hline & 5 & & 55) & $1,870(155)$ & $1,912(151)$ \\
\hline & 10 & & 61) & $3,042(152)$ & $3,174(148)$ \\
\hline & 15 & & & $3,516(125)$ & $3,660(136)$ \\
\hline
\end{tabular}

investigated. Moreover, we used objective measurements from CT scanning for the determination of working-space dimensions.

As in prior experiments, PIP and $\mathrm{ETCO}_{2}$ were shown to increase at an IAP of $10 \mathrm{mmHg}$ and higher in both groups.
However, a significant increase in MAP with $\mathrm{CO}_{2}$ insufflation was found only in group B (1st run NMB). As seen in Table 1, MAP and HR were not affected by NMB at baseline [22]. A possible explanation for the increase in MAP with abdominal $\mathrm{CO}_{2}$ insufflation in group $\mathrm{B}$ could be 
the interference of NMB with the reactions of the autonomic nervous system [23, 24].

No statistically significant effect of NMB on laparoscopic working-space dimensions was found in our porcine laparoscopy model. In contrast, there was an important effect of pre-stretching of the abdominal wall irrespective of NMB. The gain in $\mathrm{CO}_{2}$ pneumoperitoneum volume by NMB in the third insufflation-run, after two insufflationruns without NMB, could also have been caused by an extra insufflation-run and possible additional pre-stretching. The existence of such an additive effect could also explain the ever-increasing volumes with consecutive insufflation-runs, irrespective of paralysis-state or reversal of NMB, found in this study.

Pre-stretching was not facilitated by NMB, also negating its use for this purpose.

Most of the recent clinical studies [25] use deep neuromuscular block with a post-tetanic count of 1-2 responses, as opposed to the standard NMB with a TOF count of 1-2 responses (e.g., BLISS study in patients undergoing laparoscopic renal or prostatic surgery [26], CURES study in morbidly obese patients undergoing bariatric surgery [27]). Profound NMB with high dosage of rocuronium almost inevitably requires reversal of NMB at the end of the procedure. Both increase the cost of surgery [28].

In our study with non-obese pigs, we used standard NMB with a TOF of $0-1$ responses. We feel this still reflects a policy used in the majority of laparoscopic procedures in humans nowadays. Depth of anesthesia and anesthetic drugs influences muscle tone [29]. The level of anesthesia could also have influenced the effect of NMB on working space in our experiments. This correlation between depth of anesthesia and working space needs to be investigated further.

Although we could not demonstrate a positive effect of NMB on working-space dimensions, this does not negate the role of NMB in the anesthesiologic management of laparoscopic surgery. It is very effective in preventing inadvertent, sudden patient movement, which is dangerous, especially when working space is very limited as in small children.

We realize that our study uses juvenile animals as a model for a human (adult) population. Physiologic reactions to $\mathrm{CO}_{2}$ pneumoperitoneum in pigs may differ from humans [30]. Also, the anatomy of the pig's abdominal wall and pelvis differs from humans [31]. There are however enough similarities [32] to suggest the absence of a significant positive effect of NMB on laparoscopic working-space dimensions as found in this experimental and several clinical studies [6-8]. The lack of an objective method of assessing working space in humans limits clinical studies into laparoscopic working space at this time.

\section{Conclusion}

We found no evidence that NMB increases laparoscopic working space in the porcine laparoscopy model as described. Studies dealing with working space during laparoscopy should take note of pre-stretching bias.

Acknowledgments We thank L.M.E. Staals and J. Hagoort for reviewing the manuscript, M.L. Dijkshoorn for CT scanning.

Disclosures John Vlot, Patricia Specht, René Wijnen, Joost van Rosmalen, Egbert Mik, Klaas Bax have no conflict of interest or financial ties to disclose.

\section{References}

1. Emam TA et al (2000) Effect of intracorporeal-extracorporeal instrument length ratio on endoscopic task performance and surgeon movements. Arch Surg 135(1):62-65 discussion 66

2. Frede $T$ et al (1999) Geometry of laparoscopic suturing and knotting techniques. J Endourol 13(3):191-198

3. Hanna GB, Shimi S, Cuschieri A (1997) Influence of direction of view, target-to-endoscope distance and manipulation angle on endoscopic knot tying. Br J Surg 84(10):1460-1464

4. Lee AC, Haddad MJ, Hanna GB (2007) Influence of instrument size on endoscopic task performance in pediatric intracorporeal knot tying: smaller instruments are better in infants. Surg Endosc 21(11):2086-2090

5. Chassard D et al (1996) The effects of neuromuscular block on peak airway pressure and abdominal elastance during pneumoperitoneum. Anesth Analg 82(3):525-527

6. Chassard D et al (1996) Gynecologic laparoscopy with or without curare. Ann Fr Anesth Reanim 15(7):1013-1017

7. Williams MT et al (2003) A comparison of the effect of two anaesthetic techniques on surgical conditions during gynaecological laparoscopy. Anaesthesia 58(6):574-578

8. Swann DG et al (1993) Anaesthesia for gynaecological laparoscopy: a comparison between the laryngeal mask airway and tracheal intubation. Anaesthesia 48(5):431-434

9. Boon M et al (2013) Effect of variations in depth of neuromuscular blockade on rating of surgical conditions by surgeon and anesthesiologist in patients undergoing laparoscopic renal or prostatic surgery (BLISS trial): study protocol for a randomized controlled trial. Trials 14:63

10. Staehr-Rye AK et al (2013) Optimized surgical space during lowpressure laparoscopy with deep neuromuscular blockade. Dan Med J 60(2):A4579

11. Geldner $\mathrm{G}$ et al (2012) A randomised controlled trial comparing sugammadex and neostigmine at different depths of neuromuscular blockade in patients undergoing laparoscopic surgery. Anaesthesia 67(9):991-998

12. Blinman T, Ponsky T (2012) Pediatric minimally invasive surgery: laparoscopy and thoracoscopy in infants and children. Pediatrics 130(3):539-549

13. Vlot $\mathbf{J}$ et al (2013) Optimizing working space in porcine laparoscopy: CT measurement of the effects of intra-abdominal pressure. Surg Endosc 27(5):1668-1673

14. Fuchs-Buder $\mathrm{T}$ et al (2007) Good clinical research practice in pharmacodynamic studies of neuromuscular blocking agents II: the Stockholm revision. Acta Anaesthesiol Scand 51(7):789-808

15. King JWB, Roberts RC (1960) Carcass length in the bacon pig; its association with vertebrae numbers and prediction from radiographs of the young pig. Anim Prod 2(1):59-65 
16. Kim G et al (2012) Accuracy and reliability of length measurements on three-dimensional computed tomography using opensource OsiriX software. J Digit Imaging 25(4):486-491

17. Cai $\mathrm{W}$ et al (2009) MDCT for automated detection and measurement of pneumothoraces in trauma patients. AJR Am J Roentgenol 192(3):830-836

18. Neudecker $\mathbf{J}$ et al (2002) The European Association for Endoscopic Surgery clinical practice guideline on the pneumoperitoneum for laparoscopic surgery. Surg Endosc 16(7):1121-1143

19. Lindekaer AL, Halvor Springborg H, Istre O (2013) Deep neuromuscular blockade leads to a larger intraabdominal volume during laparoscopy. J Vis Exp 25(76). doi:10.3791/50045

20. Vlot J (2014) Optimizing working-space in laparoscopy: CT measurement of the effect of pre-stretching of the abdominal wall in a porcine model. Surg Endosc 28(3):841-846

21. Vlot $\mathbf{J}$ et al (2013) Optimizing working-space in laparoscopy: measuring the effect of mechanical bowel preparation in a porcine model. Surg Endosc 27(6):1980-1985

22. Levy JH et al (1994) Determination of the hemodynamics and histamine release of rocuronium (Org 9426) when administered in increased doses under N2O/O2-sufentanil anesthesia. Anesth Analg 78(2):318-321

23. Reintam Blaser A, Bloechlinger S (2010) Should we be worried about disturbed sympathovagal balance during laparoscopic cholecystectomy? Minerva Anestesiol 76(11):876-878
24. Janda $M$ et al (2013) Comparison of heart rate variability response in children undergoing elective endotracheal intubation with and without neuromuscular blockade: a randomized controlled trial. Paediatr Anaesth 23(12):1153-1159

25. National Institutes of Health (U.S.), National Library of Medicine (U.S.), and United States. Food and Drug Administration., ClinicalTrials.gov

26. Dahan A (2012) Effect of deep block on intraoperative surgical conditions (BLISS). Ongoing Clin Trial: NCT01631149

27. Vanelderen P (2012) Cures: the effect of deep curarisation and reversal with sugammadex on surgical conditions and perioperative morbidity. Clin Trial, not yet recruiting: NCT01748643

28. Ledowski $\mathrm{T}$ et al (2012) Unrestricted access to sugammadex: impact on neuromuscular blocking agent choice, reversal practice and associated healthcare costs. Anaesth Intensive Care 40(2):340-343

29. Drummond GB (2003) The abdominal muscles in anaesthesia and after surgery. Br J Anaesth 91(1):73-80

30. Richard L et al (2002) Pigs are not a reliable experimental model in the study of the haemodynamic and respiratory effects of $\mathrm{CO} 2$ pneumoperitoneum. Acta Anaesthesiol Scand 46(1):74-79

31. Swindle MM, Smith AC, Hepburn BJ (1988) Swine as models in experimental surgery. J Invest Surg 1(1):65-79

32. Srinivasan A et al (1999) Common laparoscopic procedures in swine: a review. J Invest Surg 12(1):5-14 